\title{
Punção venosa periférica: análise dos registros de acadêmicos de enfermagem
}

Peripheral venous puncture: analysis of undergraduated nursing student records

Punción venosa periférica: análisis de los registros de estudiantes de enfermeira

\section{Samylle Pelaes da Mota ${ }^{\mathrm{I}}$, Joice Silva do Nascimento ${ }^{\mathrm{II}}$, Shirlane Priscilla Barbosa de Melo Azedo $^{\text {III }}$, Camylla Cavalcante Soares de Freitas ${ }^{\mathrm{IV}}$, Alexsandra Rodrigues Feijãov ${ }^{\mathrm{V}}$ Gabriela de Sousa Martins Melo ${ }^{\mathrm{VI}}$}

Resumo: Objetivo: analisar os registros de acadêmicos de enfermagem sobre o procedimento de punção venosa periférica com cateter agulhado. Método: estudo descritivo, quantitativo, realizado com 76 acadêmicos de enfermagem do $5^{\circ}$ ao $9^{\circ}$ período de uma universidade pública, entre fevereiro e maio de 2014. Para coleta de dados desenvolveu-se cenário simulado com manequim, sendo avaliado o registro do procedimento mediante instrumento estruturado. Realizada análise descritiva. Resultados: quanto às fragilidades, 100,0\% dos alunos não realizaram o registro sobre fixação do cateter; predominou ausência da informação sobre intercorrências $(87,3 \%)$, dispositivo utilizado $(85,7 \%)$ e motivo da punção $(84,1 \%)$. Sobre os registros adequados, as variáveis data, hora e identificação sobressaíram positivamente. Conclusões: identificaram-se discrepâncias relacionadas ao registro da técnica, com ausência de informações essenciais e incompletas. Destaca-se a necessidade de trabalhar a temática em associação teoria e prática, com foco no desenvolvimento de competências no âmbito do ensino e assistência de enfermagem.

DESCRITORES: Registros de enfermagem; Estudantes de enfermagem; Avaliação em enfermagem; Cateterismo periférico

\footnotetext{
${ }^{\text {I }}$ Enfermeira. Graduada em Enfermagem pela Universidade Federal do Rio Grande do Norte (UFRN). Macapá, Amapá, Brasil. E-mail: samy_pelaes@hotmail.com ORCID iD: https://orcid.org/000-0002-3537-5406

II Enfermeira. Graduada em Enfermagem pela Universidade Federal do Rio Grande do Norte (UFRN). Natal, Rio Grande do Norte, Brasil. Email: joicesilvadonascimento@outlook.com ORCID iD: https://orcid.org/0000-0003-0469-660X

III Enfermeira. Graduada em Enfermagem pela Universidade Federal do Rio Grande do Norte (UFRN). Natal, Rio Grande do Norte, Brasil. Email: shirlaneraposo@hotmail.com ORCID iD: https://orcid.org/0000-0002-8879-6335

${ }^{\text {IV }}$ Enfermeira, Mestre em Enfermagem pela Universidade Federal do Rio Grande do Norte (UFRN). Foster City, Califórnia, Estados Unidos. Email: camyllafreitas_@hotmail.com ORCID iD: https://orcid.org/0000-0001-8508-4220

$\checkmark$ Enfermeira. Doutora em Enfermagem. Docente do Departamento de Enfermagem e do Programa de Pós-graduação em Enfermagem da Universidade Federal do Rio Grande do Norte (UFRN). Natal, Rio Grande do Norte, Brasil. E-mail: alexsandrarf@hotmail.com ORCID iD: https://orcid.org/0000-0002-8686-9502

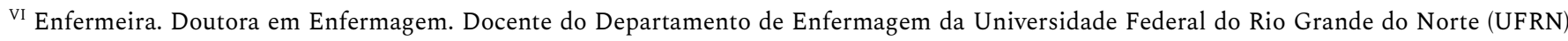
Natal, Rio Grande do Norte, Brasil. E-mail: gabrielasmm@hotmail.com ORCID iD: https:/orcid.org/0000-0002-3096-2759
} 


\begin{abstract}
Aim: To analyze undergraduated nursing students records on needle catheter peripheral venous puncture. Method: Quantitative, descriptive study, enrolled 76 undergraduated nursing students at a public university, from february through march 2014. Data collecting took place at simulated scenario and procedure records were taken with structured instrument. Descriptive analysis performed. Results: About fragilities, 100\% of the students did not make catheter fixation notes; adverse events information (87,3\%), used device (85\%) and puncture reason $(84,1 \%)$ were predominantly absent. Adequate date, time and identification records were positive highlights. Conclusion: Technique registration discrepancies were identified, with essential information absent or incomplete. Subject matter should be worked in association between theory and practice, focusing competence development at teaching environment and nursing assistance locations.
\end{abstract}

Descriptors: Nursing Records; Students, Nursing; Nursing Assessment; Catheterization, Peripheral

Resumen: Objetivo: analizar los registros de estudiantes de enfermería sobre la punción venosa periférica con catéter con aguja. Método: investigación descriptiva y cuantitativa con 76 estudiantes de enfermería de una universidad pública desde febrero hasta mayo, 2014. Para la recolección de datos, se desarrolló un escenario simulado con maniquí. Y el registro del procedimiento se evaluó con un instrumento estructurado. Se realizó el análisis descriptivo. Resultados: en cuanto a las debilidades, el 100\% de los estudiantes no registraron la fijación del catéter; predominaron la ausencia de información sobre complicaciones $(87,3 \%)$, el dispositivo utilizado $(85,7 \%)$ y el motivo de la punción $(84,1 \%)$. En cuanto a los registros apropiados, las variables fecha, hora e identificación se destacaron positivamente. Conclusiones: hubo discrepancias relacionadas con el registro de la técnica y ausencia de información esencial e incompleta. Destacamos la necesidad de trabajar el tema en asociación de la teoría y la práctica, con foco en el desarrollo de competencias en la enseñanza y la atención de enfermería.

Descriptores: Registros de Enfermería; Estudiantes de Enfermería; Evaluación en Enfermería; Cateterismo Periférico

\title{
Introdução
}

A anotação de enfermagem é um registro de informações referentes ao paciente, estruturado de forma cronológica aos acontecimentos. Seu objetivo é fornecer informações, proporcionando a comunicação da equipe e a continuidade do cuidado nas 24 horas. ${ }^{1}$ No prontuário do paciente encontram-se descritas informações que refletem o tipo de atendimento e procedimentos executados, constituindo-se como documento legal visto a necessidade de um registro claro, conciso e objetivo, além de integrar o conjunto de responsabilidades legais da enfermagem. ${ }^{2}$

A evolução de enfermagem diferencia-se da anotação, pois se refere ao registro da evolução do paciente nas últimas 24 horas, realizada exclusivamente pelo enfermeiro, uma vez que requer conhecimento técnico-científico para reflexão, análise e contextualização dos dados coletados. ${ }^{1}$

Os registros de enfermagem assumem um papel de fundamental importância no processo de cuidar. Proporcionam comunicação segura entre a enfermagem e os demais profissionais envolvidos na assistência ao paciente, contribuindo para a elaboração do plano de assistência; auxiliam na avaliação da qualidade dos serviços prestados; representam documento 
tanto para o usuário, quanto para a própria instituição; e podem ser utilizados para diversas finalidades, relacionadas ao ensino, pesquisa, processos éticos e judiciais. ${ }^{2-3}$

A Resolução do Conselho Federal de Enfermagem (COFEN) no 564/2017 Art. 36 do Código de Ética dos Profissionais de Enfermagem estabelece que é de responsabilidade da enfermagem realizar o registro no prontuário do paciente e em demais documentos pertinentes das informações inerentes e indispensáveis ao processo de cuidar, sendo dever do profissional registrar informações alusivas ao cuidado de forma clara, objetiva e completa. ${ }^{4}$

Apesar da importância do registro de enfermagem, é bastante comum observar escassez de informações, além disso, existe um descaso quanto ao processo de formalização escrita do trabalho e a falta de anotações no prontuário do paciente, o que poderia prejudicar a instituição e/ou a enfermagem em sua assistência. ${ }^{5}$

Dentre tantos procedimentos realizados na rotina de assistência da enfermagem e que exigem atenção no registro adequado, está a punção venosa periférica (PVP). A implementação da terapia endovenosa por meio da PVP é uma ação rotineira em uma instituição de saúde e, quando bem executada, auxilia na reabilitação do paciente. ${ }^{6}$ Em estudo de prevalência pontual sobre gerenciamento de cateteres intravasculares na Espanha, 81,9\% dos pacientes admitidos numa unidade hospitalar portam um ou mais cateter, destes 95,4\% de inserção periférica e curta duração. Ressalta-se a importância do domínio das técnicas visto que é um procedimento invasivo. ${ }^{7}$ Deste modo, sua utilização não está livre de complicações, sendo as mais comuns: flebite, infiltração, hematoma, trombose e tromboflebite. A PVP representa um dos procedimentos mais utilizados na assistência clínica moderna, além de ser destaque como uma das técnicas amplamente desenvolvidas pelos profissionais da área e por estudantes nas disciplinas específicas do curso de graduação em enfermagem..$^{8-9}$

A PVP é caracterizada pela instalação de um dispositivo no interior do vaso venoso, podendo ou não ser fixado à pele, e que requer cuidados e manutenção periódica, em caso de longa permanência. Ainda, exige competência técnica e destreza para sua execução, além de conhecimentos básicos de anatomia, fisiologia, microbiologia, farmacologia, psicologia, dentre outros. $^{8}$

A eficácia no desempenho para a realização da técnica de punção venosa requer conhecimentos e habilidades adquiridas no decorrer do curso mediante as atividades teóricopráticas, sendo estes requisitos básicos para os profissionais de saúde que realizam procedimentos com tal complexidade. Porém, além de destreza no desenvolvimento da técnica de PVP é necessário ainda o registro no prontuário de forma adequada.

A realização inadequada ou a não execução desses registros traz consequências na assistência ao paciente, prejuízos à instituição de saúde e dificuldades para efetuar a avaliação dos cuidados oferecidos pelos profissionais de saúde e toda equipe, uma vez que o processo de 
comunicação entre os demais profissionais que compõe uma equipe é rompido, interferindo na qualidade e segurança da assistência prestada. ${ }^{10}$

Diante dessas considerações, justifica-se a realização do presente estudo devido à importância da qualidade dos registros de enfermagem no processo de formação profissional do estudante, além disso, as considerações obtidas nesse trabalho servem como reflexão a respeito das metodologias educativas no ensino superior.

Tendo em vista a importância e a complexidade dos procedimentos executados pelos profissionais de enfermagem, surgiu a seguinte questão de pesquisa: qual a qualidade dos registros realizados por acadêmicos de enfermagem sobre a técnica de PVP? Assim, este estudo objetivou analisar os registros de acadêmicos de enfermagem sobre o procedimento de punção venosa periférica com cateter agulhado.

\section{Método}

Trata-se de um estudo descritivo, de abordagem quantitativa, realizado no Departamento de Enfermagem da Universidade Federal do Rio Grande do Norte (UFRN), no período de fevereiro a maio de 2014. A pesquisa faz parte de um projeto mestre que buscou avaliar conhecimentos e habilidades em semiologia e semiotécnica da enfermagem em ambiente simulado.

O Departamento de Enfermagem da UFRN oferece o curso de graduação em enfermagem com uma matriz curricular composta de nove períodos e está equipado com dois laboratórios de habilidades, em um dos quais foi estruturado um cenário exclusivo para esta pesquisa. Tal cenário simulado continha todos os insumos necessários para o desenvolvimento da prática de PVP com cateter agulhado, sendo o procedimento desenvolvido no simulador avançado de cuidados com o paciente adulto e gerenciamento de vias aéreas, modelo 45 CWA Simula Care ${ }^{\circledR}$, com braço específico para o desempenho da simulação da técnica, em que suas estruturas assemelham-se com a rede venosa humana, além de possibilitar o uso de sangue artificial.

Os alunos foram convidados a participar da prática simulada com manequins e para ter essa participação efetivada deveriam satisfazer os seguintes critérios de inclusão: encontrar-se regularmente matriculado no curso de Graduação em Enfermagem da UFRN e presentes no Departamento de Enfermagem durante a coleta de dados; ter cursado a disciplina de semiologia e semiotécnica da enfermagem. E, como critério de exclusão, aqueles acadêmicos que não concluíram todo o processo da coleta de dados.

Amostra sistemática, que buscou atingir 50\% de cada período. Para seleção dos participantes organizou-se as listas de frequências das turmas em ordem alfabética e numeradas de todos os matriculados no primeiro semestre de 2014. Os acadêmicos com números impares participaram da coleta referente ao procedimento de PVP. Assim, 76 alunos fizeram parte da 
amostra. Justifica-se a inclusão de metade de cada período, uma vez que os demais alunos realizaram outros procedimentos de enfermagem associados ao projeto mestre anteriormente citado.

Foi criado um instrumento de avaliação do registro de enfermagem sobre PVP, o qual é composto pelos seguintes itens fundamentais: data; hora; motivo da punção; local da punção; dispositivo utilizado; administração; fixação; intercorrências; identificação do responsável e do registro profissional ou assinatura como aluno, conforme as recomendações do Guia de recomendações para registros de enfermagem no prontuário do paciente, e do Conselho Federal de Enfermagem, sobre o registro da técnica de PVP. ${ }^{1-3}$ Ademais, coletou-se informações do perfil sociodemográfico e de experiência profissional mediante questionário aplicado previamente à simulação.

No cenário simulado desenvolvido para coleta de dados estavam disponíveis o prontuário do paciente fictício contendo a evolução de enfermagem, prescrição médica com medicamentos e condutas a serem realizadas, e impresso para registro de enfermagem, bem como todos os insumos necessários para o desenvolvimento da prática. A prescrição médica trazia dois medicamentos que deveriam ser administrados e, como parte da técnica avaliada, o aluno deveria executar a anotação do procedimento realizado na folha de registro.

Os dados coletados foram organizados em planilhas do software Microsoft Excel ${ }^{\circledR} 2010$ e realizada análise descritiva, com uso de frequência relativa e absoluta, sendo os resultados apresentados em formato de tabela e gráfico.

O estudo obteve aprovação do Comitê de Ética em Pesquisa do Hospital Universitário Onofre Lopes, sob o protocolon³85/09 (CAAE n⿳0 0002.0.294.000-10), sendo solicitada autorização da chefia do Departamento de Enfermagem UFRN para a coleta de dados e uso do laboratório de habilidades. Em tempo, todos os alunos que se encaixavam nos critérios de inclusão do estudo e que aceitaram participar voluntariamente, assinaram o Termo de Consentimento Livre e Esclarecido.

\section{Resultados}

Do total de 76 acadêmicos de enfermagem que realizaram a prática simulada de PVP com cateter agulhado, $70(92,1 \%)$ eram do sexo feminino e $63(82,9 \%)$ solteiros. Quanto ao período no curso, 16 (21,0\%) eram alunos do 5o período; $17(22,4 \%)$ do 6ㅜ; 13 (17,1\%) do 7o; $17(22,4 \%)$ do 8º; e $13(17,1 \%)$ do $9^{\circ}$ período. Relativo à experiência anterior em saúde, 13(17,1\%) tiveram alguma experiência anterior à graduação, 10 (13,2\%) tinham formação como técnico de enfermagem e 5 $(6,6 \%)$ já trabalharam na área de saúde.

Considerando os 76 participantes, 63 alunos realizaram algum registro (88,2\%), destes 59 $(93,7 \%)$ realizaram no formato de anotação de enfermagem no prontuário e somente $22(34,9 \%)$ registram a checagem na prescrição médica. No que se diz respeito à presença do registro por 
período no curso, conforme apresentado na Figura 1, destacaram-se os alunos do 6 período que em $100,0 \%$ das simulações realizaram o registro de enfermagem, e com menor porcentagem de adequação aqueles do $8^{\circ}$ período $13(76,5 \%)$.

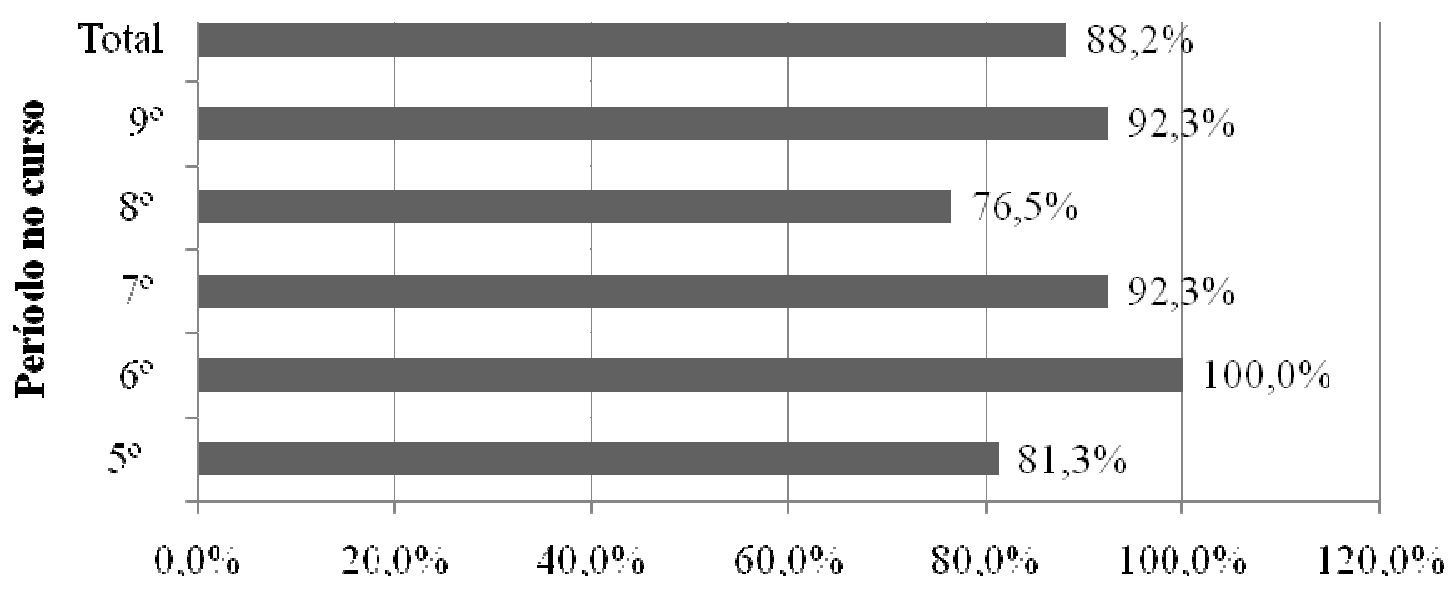

\section{Porcentagem de registro}

Figura 1 - Porcentagem de alunos que realizaram registro de enfermagem sobre PVP por período do curso. Natal, RN, Brasil, 2014

Para avaliação da qualidade dos registros, no tocante aos itens fundamentais a serem elencados (Tabela 1), foram considerados os acadêmicos que realizaram o registro na forma escrita em prontuário. Em relação aos registros adequados, o percentual foi bastante variável, os quesitos data, hora e identificação se destacaram positivamente. As variáveis do instrumento de avaliação, que são itens fundamentais para um bom registro de enfermagem sobre a PVP revelaram uma discrepância entre o registro adequado, incompleto ou ausente referente ao procedimento de PVP.

Discrepâncias no sentido de uma mesma variável ser registrada de forma adequada em porcentagem baixa e também registrada de forma incompleta em uma porcentagem alta, por exemplo. Em relação aos dados não registrados, nenhum aluno realizou o registro sobre a fixação do cateter. Também prevaleceu a ausência de informação a respeito do motivo da punção 53 (84,1\%); o dispositivo utilizado 54(85,7\%), e a presença de intercorrências 55(87,3\%).

Tabela 1 - Variáveis de identificação do registro de punção venosa periférica entre acadêmicos de enfermagem ( $\mathrm{n}=63)$. Natal, RN, Brasil, 2014

\begin{tabular}{lccccccc}
\hline \multirow{2}{*}{ VARIÁVEIS } & \multicolumn{2}{c}{$\begin{array}{c}\text { Registro } \\
\text { adequado }\end{array}$} & \multicolumn{2}{c}{$\begin{array}{c}\text { Registro } \\
\text { incompleto }\end{array}$} & \multicolumn{2}{c}{ Não registrou } \\
\cline { 2 - 8 } & $\mathbf{n}$ & $\mathbf{\%}$ & $\mathbf{n}$ & $\mathbf{\%}$ & $\mathbf{n}$ & $\mathbf{\%}$ \\
\hline Data & 62 & 98,4 & 0 & 0,0 & 1 & 1,6 \\
Hora & 52 & 82,5 & 0 & 0,0 & 11 & 17,5
\end{tabular}




\begin{tabular}{lcccccc} 
Motivo da punção & 10 & 15,9 & 0 & 0,0 & 53 & 84,1 \\
Local da punção & 17 & 27,0 & 0 & 0,0 & 46 & 73,0 \\
Dispositivo utilizado & 9 & 14,3 & 0 & 0,0 & 54 & 85,7 \\
Administração & 19 & 30,2 & 43 & 68,3 & 1 & 1,6 \\
Fixação & 0 & 0,0 & 0 & 0,0 & 63 & 100,0 \\
Intercorrências & 8 & 12,7 & 0 & 0,0 & 55 & 87,3 \\
Identificação & 42 & 66,7 & 18 & 28,6 & 3 & 4,8 \\
\hline
\end{tabular}

Considerando os nove itens avaliados na tabela 1, destaca-se que nenhum dos 63 alunos que realizaram o registro no prontuário o fez de forma completa, não havendo registro adequado de todos os itens. O mínimo de adequação foi de 1 item, máximo de 8 , média de 3,5 (desvio padrão 1,5) e mediana de 3 itens, ambos abaixo de um índice de $50 \%$ de adequações $(4,5$ itens).

Na simulação foram prescritos dois medicamentos, para o registro de administração dos mesmos o aluno precisava seguir alguns itens (nome dos medicamentos e respectivas doses de cada) para estar em conformidade com o preconizado, sendo evidenciados registros incompletos em 43 (68,3\%) das situações, 19 (30,2\%) alunos realizaram o registro adequadamente e apenas 1 $(1,6 \%)$ não realizou. As não conformidades observadas para essa variável foram: registros em quem o aluno não informou o medicamento e a dose 26 (60,5\%); não informou a dose $9(20,9 \%)$ e informou apenas um medicamento $8(18,6 \%)$.

Para o registro da variável identificação, considerando os 18 alunos que realizaram de forma incompleta, as não conformidades observadas foram: o estudante não identificou a categoria profissional ou ser acadêmico $1(5,6 \%)$ e não informou o registro do órgão de classe Conselho Regional de Enfermagem (COREN) 17 (94,4\%), quando ele se identificava como profissional enfermeiro fictício.

Observou-se também que 26 (41,3\%) dos registros os alunos deixaram espaços em branco, $11(17,5 \%)$ continha alguma rasura e $1(1,6 \%)$ algum tipo de erro de grafia.

\section{Discussão}

A enfermagem na sua atuação junto à equipe multiprofissional é quem mais se encontra incorporada no processo de cuidado, assim permanecendo por mais tempo ao lado dos pacientes, executando diversas atribuições como gerenciamento, intervenções, procedimento, em seu processo de trabalho. Com relação ao perfil dos acadêmicos pesquisados, o predomínio para o sexo feminino era presumível, seguindo a característica de feminização da profissão, corroborada pela pesquisa do perfil da enfermagem no Brasil que destaca um corpo profissional formado predominantemente pelo sexo feminino $(85,1 \%)$, contudo demonstrando uma tendência continua de crescimento da proporção do sexo masculino $(14,4 \%) .{ }^{11}$ 
Punção venosa periférica: análise dos registros de acadêmicos de enfermagem I 8

O registro é um meio de documentar toda assistência prestada tornando-a visível, e quando realizada de forma incompleta demonstra fragilidade quanto à qualidade dos cuidados. ${ }^{2}$ Um registro que apresente rasuras ou alterações, pode causar suspeitas de tentativa de alteração ou ocultação de informações. ${ }^{12}$

A PVP é um procedimento geralmente executado pela enfermagem. Um estudo realizado com estudantes da graduação na Suécia, sobre o conhecimento e habilidades da PVP com uso de manequins e auto treinamento, demonstrou que grande parte tem o domínio da técnica. ${ }^{13}$ No entanto, no que diz respeito ao registro da prática, alunos da graduação em enfermagem de uma universidade estadual relatam ter dificuldade em realizar uma anotação em prontuário, sentindo-se inseguros por não saber identificar aspectos fundamentais e relevantes. ${ }^{14}$

$\mathrm{Na}$ anotação de enfermagem, um dos itens que primeiro é descrito refere-se à data e a hora do procedimento. No estudo em questão quase todos os acadêmicos registraram a data, com exceção de um e 11 não registraram a hora que o procedimento foi realizado. Resultado semelhante foi encontrado em um estudo sobre qualidade dos registros de enfermagem em um hospital universitário, no qual a informação da data esteve presente em $80,9 \%$ dos registros do profissional enfermeiro, enquanto a hora foi menos registra com 65,5\%; enquanto que entre técnico/auxiliar de enfermagem esse índice obteve discrepância, sendo que em 94,3\% dos registros não tinham a data e $52,7 \%$ não apresentavam o horário. ${ }^{2}$

Estudos demonstram divergências com relação ao registro, em comparação com o presente estudo. Pesquisa que realizou análise dos registros de enfermagem em um hospital com certificação de acreditação hospitalar identificou que em $79 \%$ dos prontuários havia ausência da informação autor, data e hora nas anotações realizadas. ${ }^{15}$ A presença das informações corretas de data e horário (hora e minutos)são fundamentais para que os registros tenham valor legal, assim como para evitar ambiguidades, devendo toda anotação ser precedida de tais itens, inclusive a PVP. ${ }^{1}$

Um dado relevante a ser considerado, diz respeito à fixação do cateter e a importância da informação. No entanto, nenhum acadêmico realizou o registro desse momento. Destaca-se a necessidade da estabilização do cateter venoso periférico para manutenção da integridade do acesso, com prevenção da movimentação com dano ao vaso e consequente perda do dispositivo, associado ao uso de coberturas adequadas para proteger o sítio de inserção e minimizar a possibilidade de infecção, o que influencia na redução do risco de complicações como flebite, infiltração e deslocamento do cateter venoso periférico. ${ }^{16}$

Quanto à variável motivo da punção e local de punção, ambos predominaram a não realização do registro. A finalidade e o tempo de permanência do acesso venoso podem influenciar na escolha local da punção, o tipo de dispositivo e sua fixação. Geralmente, as veias superficiais e artérias periféricas dos membros superiores são mais utilizadas para o uso intermitente de medicações. ${ }^{17}$ 
Um estudo, em nível hospitalar com 76 pacientes, identificou dentre os registros realizados que $76,3 \%$ apresentavam-se completos quanto ao local de punção, descrição e calibre do dispositivo utilizado. ${ }^{18} \mathrm{O}$ tipo de dispositivo foi um dado que obteve alto percentual de ausência de anotação no presente estudo, revelando a necessidade de aprimorar essa habilidade associada ao quesito apresentado.

Em relação ao registro das intercorrências, poucos acadêmicos o fizeram, prevalecendo a ausência desse item. O uso de acesso venoso encontra-se frequentemente associado ao risco de complicações que podem conduzir ao aumento da morbidade e hospitalização prolongada, uma vez que a contaminação de cateter venoso é uma das principais causas de infecções da corrente sanguínea. ${ }^{10}$

Ainda, o Art. 38 do Código de Ética dos Profissionais de Enfermagem, traz como dever do profissional apresentar informações completas e fidedignas, seja de forma escrita ou verbal, fundamentais à continuidade do cuidado e segurança do paciente. ${ }^{4}$ Então, de acordo com o guia de recomendações para os registros de enfermagem é necessário realizar o registro das intercorrências e providências adotadas. ${ }^{3}$

A ausência de informações nos registros referentes às punções venosas impossibilita o profissional de identificar o tempo de permanência do cateter, que está diretamente relacionado com a ocorrência de complicações infecciosas. Estudo mostrou que o tempo de permanência superior a 72 horas é o fator de risco para flebite ${ }^{9}$, contrapondo as orientações da US Centers for Disease Control guidelines que indica como período ideal de manutenção para cateter periférico de 72 e 96 horas, sendo desnecessário a troca caso não seja identificado sinais flogísticos. ${ }^{19}$

O registro de administração do medicamento foi o item com mais anotações incompletas por parte dos discentes. A anotação correta do procedimento integra os “nove certos" para a administração segura de medicamentos nos pacientes. Conforme o Guia para preparo, administração e monitoramento, publicado pelo do COREN-SP em 2017, todo profissional de saúde, ao administrar um medicamento, deve sempre checar, os "nove certos": medicamento certo, dose certa, via certa, horário certo, paciente certo, registro certo, ação certa, forma certa e resposta certa. ${ }^{20}$

O último item avaliado foi a identificação, mesmo que a maioria dos acadêmicos tenha realizado esse tópico, três não o fizeram e 13 registraram de forma incompleta. Estudo de qualidade realizado com 225 prontuários apresentou inconformidade relacionados a esse dado no ato do registro de enfermagem. ${ }^{15}$

Conforme a Resolução COFEN no 429/2012 que dispõe sobre o registro das ações profissionais no prontuário do paciente, e em outros documentos próprios da enfermagem, independente do meio de suporte, é obrigatória a presença de identificação profissional e a assinatura do responsável. ${ }^{21}$ 
No que se refere ao registro adequado da técnica de PVP, o presente estudo evidenciou, ao comparar a porcentagem de anotações adequadas, incompletas ou ausentes, discrepâncias no processo. Quanto aos nove itens fundamentais para uma anotação sobre PVP, nenhum acadêmico a realizou de forma completa, fato preocupante confirmado pela baixa média e mediana de adequações. Ressalta-se a importância do registro de enfermagem de qualidade e completo para continuidade do cuidado, segurança do paciente, além de questões legais.

Por meio de uma pesquisa com dois hospitais, um público e outro privado, foram avaliados 843 registros de enfermagem com a sistematização da assistência de enfermagem (SAE), foram observados que no hospital público $42 \%$ das anotações foram feitas de forma incompleta por não apresentar "o que foi observado", "materiais utilizados" bem como as "intercorrências clínicas" inerentes ao procedimento. No entanto, no hospital particular $81 \%$ das informações escritas no prontuário apresentaram os dados supracitados completos. Além disso, os hospitais foram classificados quanto os seus registros em satisfatório e insatisfatório considerando todas as informações neles contido, sendo os do público denominados insatisfatório (71\%) e os do particular satisfatório (81\%). ${ }^{22}$

O registro de enfermagem é uma prática essencial no cotidiano da equipe de enfermagem, por comprovar todos os cuidados prestados pelos profissionais responsáveis, com fidelidade e legitimidade ao cenário do cuidar. Sua omissão inviabiliza a comunicação multiprofissional a respeito da situação do paciente. ${ }^{2}$

Trata-se de um documento que quando expressa qualidade representa significativamente o cuidado de enfermagem, porém quando inexistente, com lacunas resulta em uma série de problemas comprometendo a assistência e continuidade do cuidado. ${ }^{2}$ Acredita-se que os itens do procedimento PVP, cujos índices de dados incompletos ou ausentes foram significativos, merecem ser revistos no âmbito do ensino e da assistência de enfermagem.

Frente às diversas competências necessárias para formação do enfermeiro, a ansiedade e insegurança estão relacionadas ao contexto das relações e demandas acadêmicas. Devido a fatores como sobrecarga, exposição a momentos de tensão, estresse, expectativas do mercado, atividades práticas e estágios, cobrança nos estudos, lidar com situações desconhecidas, como a realização de um procedimento novo ou cuidado direto ao paciente. ${ }^{23}$ Tais anseios podem ser minimizados com uso de metodologia ativa, tornando o processo de ensino-aprendizagem mais eficiente e interativo.

Nesse contexto, a simulação realística destaca-se como estratégia de ensino capaz proporcionar o desenvolvimento de habilidades, mediante experiências reproduzindo aspectos e situações da realidade de forma fictícia e segura, com o auxílio de atores ou uso de manequins, em cenários previamente estabelecidos, permitindo a melhora do desempenho dos participantes a partir dos seus próprios erros; além de trabalhar habilidades técnicas, possibilita o exercício da liderança, trabalho em equipe e o raciocínio crítico. ${ }^{24}$ 
Assim, as transformações que acontecem na sociedade contemporânea estabelecem desafios para as instituições formadoras. As transformações valorizam o processo formativo que propicia uma visão holística do ser humano e sociedade, para intervir em suas problemáticas, de forma problematizadora, libertadora, superando a dicotomia entre teoria e prática, demandando maior necessidade de qualificação e reflexão crítica dos profissionais de enfermagem. ${ }^{25}$

A presença de componentes específicos profissionalizantes que trabalham o conhecimento e a execução de técnicas como a PVP de forma segmentada, dificultam a consolidação do conhecimento e das habilidades necessárias para procedimento. A fragmentação no processo formativo torna-se um fator dificultador para sedimentação do aprendizado, sendo imperativo o desenvolvimento de um perfil de continuidade, transformando o aprendizado em algo contínuo, efetivo e consciente.

\section{Conclusão}

O presente estudo identificou que foram frequentes as discrepâncias relacionadas ao registro da técnica de punção venosa com cateter agulhado por acadêmicos de enfermagem como, anotações incompletas e ausência de informações. Os quesitos data, hora e identificação se destacaram positivamente, com maiores índices de adequação.

Não houve nenhum registro sobre a fixação do cateter. Também prevaleceu a ausência de informação sobre o motivo da punção, dispositivo utilizado e a presença de intercorrências. Destaca-se que dos itens avaliados, nenhum acadêmico realizou a anotação do procedimento de forma adequada e completa, sendo a média de adequação baixa (3,5 itens).

Como fragilidade do estudo destacam-se a impossibilidade de associação entre os participantes que realizaram a checagem da prescrição médica e a anotação do procedimento no prontuário, assim como a dificuldade de relação entre a anotação e quem a executou, uma vez que cada participante poderia assinar de forma fictícia e a folha do prontuário não era associada à identificação do acadêmico, gerando dificuldades para análises mais aprofundadas, especialmente no que tange a comparação entre os diferentes períodos.

Para melhores resultados em estudos futuros que contemplem essa temática, recomendase a rever os conhecimentos desse processo no âmbito do ensino e da assistência de enfermagem, como também trabalhar o processo formativo e avaliativo durante o curso, buscando identificar as estratégias de formação e acompanhamento dos acadêmicos quanto ao procedimento, assim como para demais habilidades necessárias ao enfermeiro.

É necessário trabalhar mais a temática de forma associada entre a teoria e a prática, uma vez que os acadêmicos serão futuros profissionais e disseminadores de conhecimentos, o que poderá repercutir na qualidade do egresso e, consequentemente, da assistência de enfermagem. A partir da identificação dos pontos de melhoria, pode-se atuar buscando fornecer subsídios 
para progresso do ensino, além de fomentar pesquisas na enfermagem que incentivem a discussão nesta área, refletindo sobre o processo de ensino-aprendizagem, uso de metodologias ativas e recursos voltados para o aperfeiçoamento dos enfermeiros em formação.

\section{Referências}

1. Conselho Regional de Enfermagem de São Paulo (COREN-SP). Anotações de Enfermagem. São Paulo (SP); 2009.

2. Diniz SOS, Silva PS, Figueredo NMA, Tonini T. Qualidade dos registros de enfermagem: reflexões analíticas em suas formas e conteúdos. Rev Enferm UFPE On Line [Internet]. 2015 [acesso em 2017 nov 07];9(10):9616-23. Disponível em: https://periodicos.ufpe.br/revistas/revistaenfermagem/article/view/10908

3. Conselho Federal de Enfermagem (COFEN). Guia de recomendações para registro de enfermagem no prontuário do paciente e outros documentos de enfermagem. Brasília (DF): COFEN; 2016.

4. Conselho Federal de Enfermagem (COFEN). Resolução COFEN n. 564/2017. Aprova novo código de ética dos profissionais de enfermagem. Rio de Janeiro (RJ): COFEN; 2017.

5. Oliveira AKA, Vasconcelos QLDAQ, Melo GSM, Melo MDM, Costa IKF, Torres GV. Validação de instrumento para punção venosa periférica com cateter agulhado. Rev Rene [Internet]. 2015 [acesso em 2017 nov 07];16(2):176-84. Disponível em: http://periodicos.ufc.br/rene/article/view/2701

6. Frota NM, Barros LM, Araújo TM, Caldini LN, Nascimento JC, Caetano JA. Construção de uma tecnologia educacional para o ensino de enfermagem sobre punção venosa periférica. Rev Gaúch Enferm [Internet]. 2013 [acesso em 2017 maio 23];34(2):29-36. Disponível em: http://www.scielo.br/scielo.php?script=sci_arttext\&pid=S1983-14472013000200004\&lng=en

7. Guembe M, Pérez-Granda MJ, Capdevila JA, Barberán J, Pinilla B, Martín-Rabadán P, et al. Nationwide study on the use of intravascular catheters in internal medicine departments. J Hosp Infect [Internet]. 2015 [acesso em 2017 nov 07];90(2):135-41. Disponível em: https://www.ncbi.nlm.nih.gov/pubmed/25824558

8. Oliveira AKA, Medeiros LP, Melo GSM, Torres GV. Passos da técnica de punção venosa periférica: revisão integrativa. Arq Ciênc Saúde [Internet]. 2014 [acesso em 2017 maio 23];21(1)88-95. Disponível em: http://repositorio-racs.famerp.br/racs_ol/vol-21-1/v21-1.htm

9. Johann DA, Danski MTR, Vayego SA, Barbosa DA, Lind J. Fatores de risco para complicações no cateter venoso periférico em adultos: análise secundária de ensaio clínico randomizado. Rev Latinoam Enferm [Internet]. 2016 [acesso em 2017 nov 07];24:e2833. Disponível em: http://www.redalyc.org/articulo.oa?id=281449727056 
10. Silva BF, Lopes FA, Queiroz TQ, Sousa TDL, Gomes RKG. Inovações na segurança do paciente assistido em clínica-médica: qualidade dos registros da equipe de saúde nos prontuários. Mostra Interdiscip Curso Enferm [Internet]. 2016 [acesso em 2017 nov 07];02(2). Disponível em: http://publicacoesacademicas.unicatolicaquixada.edu.br/index.php/mice/index

11. Machado MH, Aguiar WF, Lacerda WF, Oliveira E, W Lemos, Wermelinger M, et al. Relatório final da pesquisa perfil da enfermagem no Brasil. Rio de Janeiro (RJ): Fiocruz; 2015.

12. Caveião C, Hey AP, Montezelli JH, Gastandi AB, Bobroff MCC, Farias DS. Anotações de enfermagem na perspectiva de auxiliares de enfermagem. Rev Enferm UFSM [Internet]. 2014 [acesso em 2017 junho 12];4(2):401-9. Disponível em: https://periodicos.ufsm.br/reufsm/article/view/10720/pdf

13. Ahlin C, Klang-Söderkvistet B, Johansson E, Björkholmal M, Löfmark A. Assessing nursing students' knowledge and skills in performing venepuncture and inserting peripheral venous catheters. Nurse Educ Pract [Internet]. 2017 [acesso em 2017 nov 12];23:8-14. Disponível em: https://www.ncbi.nlm.nih.gov/pubmed/28171853

14. Silva JP, Garanhani ML, Peres MA. Sistematização da assistência de enfermagem na graduação: um olhar sob o pensamento complexo. Rev Latinoam Enferm [Internet]. 2015 [acesso em 2017 nov 07];23(1):59-66. Disponível em: http://www.redalyc.org/articulo.oa?id=281438429009

15. Morais CGX, Batista EMS, Castro JFL, Assunção SS, Castro GMO. Registros de enfermagem em prontuário e suas implicações na qualidade assistencial segundo os padrões de acreditação hospitalar: um novo olhar da auditoria. Rev ACRED [Internet]. 2015 [acesso em 2017 maio 07];5(9):64-84. Disponível em: https://dialnet.unirioja.es/servlet/articulo?codigo $=5626617$

16. Agência Nacional de Vigilância Sanitária (ANVISA). Medidas de prevenção de infecção da corrente sanguínea. Brasília (DF): Anvisa; 2017. Medidas de Prevenção de Infecção Relacionada à Assistência à Saúde; p. 49-70.

17. Soares CR, Almeida AM, Gozzo TO. A avaliação da rede venosa em mulheres com câncer ginecológico durante o tratamento quimioterápico. Esc Anna Nery Rev Enferm [Internet]. 2012 [acesso em 2017 maio 23];16(2):240-6. Disponível em: http://www.scielo.br/scielo.php?script=sci_arttext\&pid=S1414-81452012000200005\&lng=en

18. Tertuliano AC, Borges JLS, Fortunato RAS, Oliveira AL, Poveda VB. Flebite em acessos venosos periféricos de pacientes de um Hospital do Vale do Paraíba. REME Rev Min Enferm [Internet]. 2014 [acesso em 2017 maio 23];18(2):334-45. Disponível em: http://reme.org.br/artigo/detalhes/931

19. Webster J, Osborne S, Rickard CM, New K. Clinically-indicated replacement versus routine replacement of peripheral venous catheters. Cochrane Database Syst Rev [Internet]. 2015 [acesso em 2017 maio 07];14:CD007798. Disponível em: https://www.cochranelibrary.com/cdsr/doi/10.1002/14651858.CD007798.pub4/full 
20. Conselho Regional de Enfermagem de São Paulo. Uso seguro de medicamentos: guia para preparo, administração e monitoramento. São Paulo (SP): COREN-SP, 2017.

21. Conselho Federal de Enfermagem (COFEN). Resolução COFEN n. 429/2012. Dispõe sobre o registro das ações profissionais no prontuário do paciente, e em outros documentos próprios da enfermagem, independente do meio de suporte - tradicional ou eletrônico. Brasília (DF): COFEN; 2012.

22. Silva TG, Santos RM, Crispim LMC, Almeida LMWS. Conteúdo dos registros de enfermagem em hospitais: contribuições para o desenvolvimento do processo de enfermagem. Enferm Foco [Internet]. 2016 [acesso em 2017 nov 23];7(1):24-27. Disponível em: http://revista.cofen.gov.br/index.php/enfermagem/article/view/679

23. Jesus IS, Sena ELS, Souza LS, Pereira LC, Santos VTC. Vivências de estudantes de graduação em enfermagem com a ansiedade. Rev Enferm UFPE On Line [Internet]. 2015 [acesso em 2018 set 03];9(1):149-57. Disponível em: https://periodicos.ufpe.br/revistas/revistaenfermagem/article/download/10319/11001

24. Shapira-Lishchinsky O. Simulations in nursing practice: toward authentic leadership. J NursManag [Internet]. $2014 \quad$ [acesso em 2018 set 05];22(1):60-9. Disponível em: https://www.ncbi.nlm.nih.gov/pubmed/23410036

25. Pires AS, Souza NVDO, Penna LHG, Tavares KFA, D'Oliveira CAFB, Almeida CM. A formação de enfermagem na graduação: uma revisão integrativa da literatura. Rev Enferm UERJ [Internet]. 2014 [acesso em 2018 set 05];22(5):705-11. Disponível em: http://www.epublicacoes.uerj.br/index.php/enfermagemuerj/article/view/11206/12315

\section{Autor correspondente:}

Gabriela de Sousa Martins Melo

E-mail: gabrielasmm@hotmail.com

Endereço: Campus Universitário, BR 101 - Lagoa Nova. Natal/ RN

CEP: $59072-970$

\section{Contribuições de Autoria}

1 - Samylle Pelaes da Mota

Autora realizou o projeto de pesquisa, destaca-se que o estudo faz parte do seu trabalho de conclusão de curso em enfermagem da UFRN, participou de todo planejamento, execução e confecção do manuscrito.

2 - Joice Silva do Nascimento

Contribuições: Autora participou da tabulação, análise e interpretação dos dados e redação do texto.

3 - Shirlane Priscilla Barbosa de Melo Azedo Raposo

Autora participou da tabulação, análise e interpretação dos dados e redação do texto. 
15 I Mota SP, Nascimento JS, Azedo SPBM, Freitas CCS, Feijão AR, Melo GSM

4 - Camylla Cavalcante Soares de Freitas

Autora participou como auxiliar de pesquisa, ajudando na obtenção dos dados, redação e revisão crítica do manuscrito.

5 - Alexsandra Rodrigues Feijão

Autora contribuiu com redação e revisão critica do manuscrito.

6 - Gabriela de Sousa Martins Melo:

Pesquisadora responsável e orientadora do trabalho, fazendo parte da concepção e planejamento do projeto de pesquisa, obtenção e análise dos dados, além de participar da revisão crítica do estudo.

\section{Como citar este artigo}

Mota SP, Nascimento JS, Azedo SPBM, Freitas CCS, Feijão AR, Melo GSM. Punção venosa periférica: análise dos registros de acadêmicos de enfermagem. Rev. Enferm. UFSM. 2019 [Acesso em: Anos Mês Dia]; vol e2:1-14. DOI:https://doi.org/10.5902/2179769230148 